\title{
Revisores 2013
}

El Equipo Editorial desea agradecer, muy sinceramente, el trabajo realizado por los revisores. Somos conscientes del esfuerzo y el tiempo que ello supone.

Abdulla, Abel Zagazig University. Egipto.

Alegre, Alegre. East Stroudsburg University. EEUU.

Alkharusi, Hussain. Sultan Cabos University. Omán

Álvarez, Ibis Marlene. Universitat Autonòma de Barcelona. España

August, Diane. Centre for Appled Linguistiques, Washington. EEUU.

Avilar, Jose María. Universidad de Valladolid. España

Barberá, Elena. Universidad de Barcelona. UOC. España

Bartels, Jared M.. University of Central Missouri. EEUU

Bermúdez, José. UNED, Madrid. España

Bernardo, Allan B.I. University of Macau, Macau SAR. China

Bethencourt, José Tomás. Universidad de La Laguna. España

Cano, Francisco. Universidad de Granada. España

Cardelle-Ellawar, Maria. Arizona State University. EEUU.

Chen, I’Jung. Universidad de Taipei. Taiwan

Dessoette, Annemi Ghent University. Bélgica

Enriques, Manuel J. Universidad de Lisboa. Portugal

Escoriza, José. Universidad de Barcelona. España

Fabiola Ruiz, Elena Instituto Politécnico Nacional, México

Fernández-Berrocal, Pablo. Universidad de Málaga. España

Fernández-Lozano, Pilar. Universidad Complutense de Madrid. España

Gaeta, Marta Leticia Universidad de Puebla. México

García-Bergén, Ana Belén. Universidad de Granada. España

García Ros, Rafael. Universidad de Valencia. España 
Glover, Patti. Gonzaga University. EEUU.

González, Carmen. University of Murcia. España

Granda Vera, Juan. Universidad de Granada. España

Günter Faber. Leibniz University of Hannover. Germany

Gutiérrez, Mario Fernando. Profesional Psicólogo. Colombia.

Guzel-Ozmen, G.. Gazi Universitesi, Turquía

Hintsanen, Mirka University of Helsinki. Finlandia

Hinz, Arnold. University of Education. Alemania

Inglés, Cándido. Universidad Miguel Hernández. España

Iriarte, Concha. Universidad de Navarra. España

Flores, Oscar. Universidada de Lleida. España

Jiménez, Virginia. Universidad Complutense de Madrid. España

Kaplan, Avi. Ben-Gurion University of the Negev. Israel

Krueger, Norris F. Boise State University, EEUU.

Lavigne, Rocío, Universidad de Málaga. España

Linderholm, Tracy. Georgia Southern University. EEUU.

López, Esther. UNED. Madrid. España

Martínez, Hector. Universidad Católica de Santa María. Perú.

Martínez-González, Agustín. Clínica Neuropsicológica Mayor (Alcantarilla, Murcia). España

Martínez-Vicente, J. M. Universidad de Almería. España

Mata, Sara. Universidad de Granada. España

Matero. Vicente Félix. Hospital Clínico de Valencia. España

Maherzi, Sena. Effat University. Arabia Saudí

Melville, Wayne. Lakehead University, Ontario. Canadá

Morales, Francisco Manuel. Universidad de Málaga. España

Moriana, Juan Antonio. Universidad de Córdoba. España

Muñoz, María Teresa. Universidad Católica de Maule. Chile

Ozdemir, Selda. Gazi University. Turquía

Ordaz, Gabriela. UNAM. México

Orjinés. Mireia. Universidad Miguel Hernández. Alicante. España

Papantoniou, Georgia. University of Loannina. Grecia

Peralta, Francisco J. Universidad de Almería. España

Phan, Huy P. University of the South Pacific. Islas Fiji

Polo, Tamara. Universidad de Granada. España 
Ponce, Hector. Universidad Santiago de Chile. Chile

Prieto Sánchez, $\mathrm{M}^{\mathrm{a}}$ Dolores. Universidad de Murcia. España,

Rivera, Santiago Jorge. Universidad de Pinar del Río. Cuba

Rodríguez, Antonio. Universidad de Granada. España

Sander, Paul. Cardiff Metropolitan University. Reino Unido

Suriá, Raquel. Universidad de Alicante. España

Torres, Manuela. Fonos Salud. España

Trianes, Maria Victoria. Universidad de Málaga. España

Tzivinikou, Sotiria. Aristotle University of Thessalonik. Grecia

Villegas, José Luis, Universidad de Granada. España

Walker, Stacy E.. William Paterson University, EEUU.

Yaziri, Ersen. Selçuk University, Konya. Turquía

Young, Alys. University of Manchester. Reino Unido

Valenzuela, Jorge. Université Catholique de Louvain. Bélgica

Verónica, Paola. Consejo Nacional de Investigaciones Científicas. Argentina

Villardón, Lourdes. Universidad de Deusto. España

\section{Porcentaje de aceptación- rechazo 2013}

Artículos recibidos: $109(100 \%) \quad$ Aceptados: 35\% Rechazos: 65\% 
Electronic Journal of Research in Educational Psychology

[Página en blanco por razones de paginación] 